Sudheedra Rao, M. N., 323

Swamy, M., 31

Swita. E.. 109

Tamulewicz, S., 341, 343, 345, 347

Taylor, D. K.. 491

Tejero. T.. 441. 443Telser, J.. 445

Thaker. D. A.. 13

Thimmaiah. K. N., 21, 23, 25, 27

Thomas. L. M., 57

Thöne. C.. 118

Tiekink. E. R. T., 163, 165, 167, 169, 350, $351,353,355,357,359,361,363,365$

Tillard-Charbonnel, M., 509

Tillmanns. E.. 171

Tochtermann, W., 367, 369, 371, 373, 375

Tomas. A.. 337

Toniolo. C., 113

Tomero, J., 377

Torres, M. R., 377

Trávnícek, Z., 123, 125

Trenkle, H., 467

Troll, A., 449

Tyvanchuk, Yu. B., 291
Uecker, R., 76

Ueyama, N., 325

Uhlemann, E., 65, 333

Ülki், D., 493

Valle, G., 113

Vencato, I., 41

Villacampa, M. D.. 121

Vincent, L., 509

Vindyš, M.. 459

Viossat, B., 337

Vittal, J. J., 409, 411

Wallis, A. F. A., 473, 475

Vladimirov, S., 59,61

Wacker, K., 94

Walther, R., 293

Wang, P.-L., 267

Warda, S. A., 319

Wardell, J. L., 379

Wartchow, R., 43, 80, 81, 83, 320, 439

Weber, E., 327

Webster, C. J., 256

Weinkauf, A., 119

Weirich, Th. E., 301
Welle, F. M., 163

Weller, F., 37, 157, 159, 332, 333

Wemer, P.-E., 267

White, R., 49

Whittingham, W. S., 321

Wicharz, R., 81

Wiebelhaus, D. 335

Wojnowski, W., 341, 343, 345, 347, 349

Worzala, H., 195, 197, 199, 393, 395

Wu, E. J., 91

Wu, L. Y., 101, 105

Yagbasan, R., 183, 185

Yaghi, O. M., 453, 455, 457

Yanson, T. I., 504, 505

Young, D. J., 49

Yvon, K., 289, 290, 504, 505, 508

Zachrisson, V., 45

Zahn, G., 73

Zaremba, V. I., 291

Zavalij, P. Y., 321

Ziegler, T., 205

Zivanov-Stakic, D., 59, 61

Zsoldos-Mády, V., 189

\title{
Formulae Index of Volume 212 Issues 1-3
}

\author{
$\mathrm{Ag}_{0.6} \mathrm{Al}_{3.4} \mathrm{La}, 288$ \\ $\mathrm{AgHfRbTe}_{3}, 92$ \\ $\mathrm{AgK}_{2} \mathrm{P}, 78$ \\ $\mathrm{Ag}_{3} \mathrm{KSe}_{2}, 97$ \\ $\mathrm{Al}_{0.65} \mathrm{La}_{0.27} \mathrm{O}_{3} \mathrm{~S}_{0.73} \mathrm{Ta}_{0.35}, 77$ \\ $\mathrm{AlBaF}_{6} \mathrm{Na}, 89$ \\ $\mathrm{Al}_{2} \mathrm{FeZr}_{6}, 504$ \\ $\mathrm{Al}_{3} \mathrm{EuPd}_{2}, 287$ \\ $\mathrm{Al}_{5.9} \mathrm{Fe}_{6.1} \mathrm{Lu}, 505$ \\ $\mathrm{Al}_{6} \mathrm{Cl}_{2} \mathrm{Na}_{8} \mathrm{O}_{24} \mathrm{Si}_{6}, 80$ \\ $\mathrm{Al}_{7.72} \mathrm{Fe}_{4.28} \mathrm{~Tb}, 505$ \\ $\mathrm{Al}_{8} \mathrm{Fe}_{4} \mathrm{La}, 505$ \\ $\mathrm{Al}_{8} \mathrm{Fe}_{4} \mathrm{Lu}, 505$ \\ $\mathrm{As}_{2} \mathrm{HgK}_{4}, 79$ \\ $\mathrm{AsBBa}_{3} \mathrm{O}_{3}, 300$ \\ $\mathrm{BBr}_{6} \mathrm{Y}_{4}, 99$ \\ $\mathrm{B}_{6} \mathrm{H}_{6} \mathrm{Li}_{2} \mathrm{O}_{18} \mathrm{P}_{2}, \quad 313$ \\ $\mathrm{Ba}_{9} \mathrm{~N}_{12} \mathrm{Nb}_{2}, 309$ \\ $\mathrm{Bi}_{6} \mathrm{IKO}_{9}, 88$ \\ $\mathrm{Br}_{3} \ln \mathrm{Mg}, 5$ \\ $\mathrm{Br}_{6} \mathrm{DyLiRb}_{2}, 2$ \\ $\mathrm{Br}_{13} \mathrm{Cu}_{4} \mathrm{Rb}_{3} \mathrm{Tm}_{2}, 1$ \\ $\mathrm{C}_{2} \mathrm{H}_{4} \mathrm{Cl}_{5} \mathrm{~N}_{2} \mathrm{P}_{3}, 244$ \\ $\mathrm{C}_{2} \mathrm{H}_{8} \mathrm{Br}_{3} \mathrm{NPb}, 234$ \\ $\mathrm{C}_{2} \mathrm{H}_{10} \mathrm{Cl}_{2} \mathrm{~N}_{2}, 188$ \\ $\mathrm{C}_{3} \mathrm{H}_{12} \mathrm{~F}_{4} \mathrm{MnNO}_{2}, \quad 199,393$ \\ $\mathrm{C}_{3} \mathrm{H}_{16} \mathrm{~F}_{4} \mathrm{MnNO}_{4}, 197$ \\ $\mathrm{C}_{4} \mathrm{H}_{4} \mathrm{~N}_{2} \mathrm{O}_{3}, 407$ \\ $\mathrm{C}_{4} \mathrm{H}_{9} \mathrm{BrO}_{2} \mathrm{Sn}, 117$ \\ $\mathrm{C}_{5} \mathrm{H}_{5} \mathrm{Tl}, 47$ \\ $\mathrm{C}_{5} \mathrm{H}_{6} \mathrm{I}_{3} \mathrm{NPb}, 235$ \\ $\mathrm{C}_{5} \mathrm{H}_{9} \mathrm{NO}_{4}, 226$ \\ $\mathrm{C}_{5} \mathrm{H}_{13} \mathrm{NO}_{5} \mathrm{Se}, 51$ \\ $\mathrm{C}_{5} \mathrm{H}_{18} \mathrm{~F}_{5} \mathrm{FeN}_{2} \mathrm{O}_{2}, 195$
}

\author{
$\mathrm{C}_{5} \mathrm{H}_{18} \mathrm{~F}_{5} \mathrm{FeN}_{2} \mathrm{O}_{2}, 395$ \\ $\mathrm{C}_{6} \mathrm{H}_{6} \mathrm{FeK}_{2.9} \mathrm{Na}_{0.1} \mathrm{O}_{15}, 83$ \\ $\mathrm{C}_{6} \mathrm{H}_{8} \mathrm{~N}_{2} \mathrm{O}_{4}, 429$ \\ $\mathrm{C}_{6} \mathrm{H}_{8} \mathrm{NNaO}_{5}, 169$ \\ $\mathrm{C}_{6} \mathrm{H}_{12} \mathrm{Br}_{2} \mathrm{HgN}_{4}, 154$ \\ $\mathrm{C}_{6} \mathrm{H}_{13} \mathrm{ClO}_{2} \mathrm{Sn}, 332$ \\ $\mathrm{C}_{6} \mathrm{H}_{14} \mathrm{ClNO}_{4}, 213,217$ \\ $\mathrm{C}_{6} \mathrm{H}_{16} \mathrm{~N}_{6} \mathrm{O}_{6}, 414$ \\ $\mathrm{C}_{6} \mathrm{H}_{18} \mathrm{Cl}_{4} \mathrm{O}_{3} \mathrm{~Pb}_{2} \mathrm{~S}_{3}, 236$ \\ $\mathrm{C}_{6} \mathrm{H}_{18} \mathrm{ClN}_{3} \mathrm{Zr}, 490$ \\ $\mathrm{C}_{6} \mathrm{H}_{20} \mathrm{Cl}_{4} \mathrm{CuN}_{6}, 377$ \\ $\mathrm{C}_{6} \mathrm{H}_{21} \mathrm{Cl}_{3} \mathrm{~N}_{2}, 401$ \\ $\mathrm{C}_{7} \mathrm{H}_{7} \mathrm{NO}_{4} \mathrm{~S}, 282$ \\ $\mathrm{C}_{8} \mathrm{H}_{14} \mathrm{I}_{3} \mathrm{NOPbS}, 243$ \\ $\mathrm{C}_{8} \mathrm{H}_{18} \mathrm{CO}_{2} \mathrm{O}_{17}, 81$ \\ $\mathrm{C}_{8} \mathrm{H}_{24} \mathrm{Mo}_{3} \mathrm{~N}_{2} \mathrm{~S}_{13}, 303$ \\ $\mathrm{C}_{8} \mathrm{H}_{27} \mathrm{As}_{7} \mathrm{~N}_{3} \mathrm{Rb}, 85$ \\ $\mathrm{C}_{8} \mathrm{H}_{32} \mathrm{Cl}_{10} \mathrm{~N}_{4} \mathrm{~Pb}_{3}, 241$ \\ $\mathrm{C}_{8} \mathrm{H}_{64} \mathrm{~N}_{2} \mathrm{Na}_{4} \mathrm{O}_{48} \mathrm{~V}_{10}, 321$ \\ $\mathrm{C}_{9} \mathrm{H}_{6}$ INO, 387 \\ $\mathrm{C}_{9} \mathrm{H}_{9} \mathrm{~N}_{3}, 30$ \\ $\mathrm{C}_{9} \mathrm{H}_{10} \mathrm{~N}_{3} \mathrm{O}_{2}, 415$ \\ $\mathrm{C}_{9} \mathrm{H}_{12} \mathrm{ClN}_{3} \mathrm{O}_{4}, 33$ \\ $\mathrm{C}_{9} \mathrm{H}_{15} \mathrm{~N}_{5} \mathrm{OS}_{3} \mathrm{Zn}, 155$ \\ $\mathrm{C}_{9} \mathrm{H}_{19} \mathrm{NO}_{5}, 215$ \\ $\mathrm{C}_{10} \mathrm{H}_{4} \mathrm{CeO}_{9}, 457$ \\ $\mathrm{C}_{10} \mathrm{H}_{8} \mathrm{FeSe}_{3}, 118$ \\ $\mathrm{C}_{10} \mathrm{H}_{11} \mathrm{Cl}_{3} \mathrm{CoN}_{2}, 472$ \\ $\mathrm{C}_{10} \mathrm{H}_{12} \mathrm{Cl}_{2} \mathrm{O}, 265$ \\ $\mathrm{C}_{10} \mathrm{H}_{12} \mathrm{NO}_{3} \mathrm{Se}_{4}, 101$ \\ $\mathrm{C}_{10} \mathrm{H}_{18} \mathrm{NiO}_{2} \mathrm{~S}_{4}, 350$ \\ $\mathrm{C}_{10} \mathrm{H}_{32} \mathrm{Cl}_{2} \mathrm{~N}_{6} \mathrm{Ni}_{2} \mathrm{O}_{15}, 123$ \\ $\mathrm{C}_{11} \mathrm{H}_{11} \mathrm{ClO}_{2}, 491$ \\ $\mathrm{C}_{11} \mathrm{H}_{18} \mathrm{ClNO}_{3}, 113$
}

$\mathrm{C}_{11} \mathrm{H}_{24} \mathrm{ClN}_{5} \mathrm{O}_{2}, 129$

$\mathrm{C}_{12} \mathrm{H}_{8} \mathrm{Cs}_{4} \mathrm{~N}_{12} \mathrm{O}_{4} \mathrm{Re}_{4} \mathrm{Te}_{4}, 308$

$\mathrm{C}_{12} \mathrm{H}_{10} \mathrm{~N}_{2} \mathrm{O}, 283$

$\mathrm{C}_{12} \mathrm{H}_{12} \mathrm{O}_{2}, 256$

$\mathrm{C}_{12} \mathrm{H}_{13} \mathrm{NO}_{3}, 419$

$\mathrm{C}_{12} \mathrm{H}_{14} \mathrm{Cl}_{2} \mathrm{CoN}_{2}, 41$

$\mathrm{C}_{12} \mathrm{H}_{15} \mathrm{NO}_{5}, 417$

$\mathrm{C}_{12} \mathrm{H}_{15} \mathrm{NO}_{7}, 53$

$\mathrm{C}_{12} \mathrm{H}_{18} \mathrm{~N}_{4} \mathrm{O}_{4}, 163$

$\mathrm{C}_{12} \mathrm{H}_{22} \mathrm{Cl}_{2} \mathrm{O}_{4} \mathrm{Pt}_{2}, \quad 181$

$\mathrm{C}_{12} \mathrm{H}_{23} \mathrm{NaO}, 329$

$\mathrm{C}_{12} \mathrm{H}_{25} \mathrm{~N}_{3} \mathrm{O}_{6}, 233$

$\mathrm{C}_{12} \mathrm{H}_{28} \mathrm{I}_{3} \mathrm{NPb}, 237$

$\mathrm{C}_{12} \mathrm{H}_{28} \mathrm{NO}_{3}, 229$

$\mathrm{C}_{12} \mathrm{H}_{28} \mathrm{O}_{4} \mathrm{~V}, 485$

$\mathrm{C}_{13} \mathrm{H}_{8} \mathrm{Cl}_{2} \mathrm{O}, 339$

$\mathrm{C}_{13} \mathrm{H}_{9} \mathrm{BrO}, 339$

$\mathrm{C}_{13} \mathrm{H}_{14} \mathrm{O}_{4}, 423$

$\mathrm{C}_{13} \mathrm{H}_{14} \mathrm{O}_{6}, 45$

$\mathrm{C}_{13} \mathrm{H}_{19} \mathrm{NO}_{9} \mathrm{~S}, 427$

$\mathrm{C}_{14} \mathrm{H}_{8} \mathrm{~N}_{2}, 100$

$\mathrm{C}_{14} \mathrm{H}_{13} \mathrm{NO}_{3}, 29$

$\mathrm{C}_{14} \mathrm{H}_{14} \mathrm{~N}_{2} \mathrm{O}_{3} \mathrm{~S}, 277$

$\mathrm{C}_{14} \mathrm{H}_{16} \mathrm{BrN}, 200$

$\mathrm{C}_{14} \mathrm{H}_{16} \mathrm{O}_{5} \mathrm{P}_{2}, 119$

$\mathrm{C}_{14} \mathrm{H}_{18} \mathrm{~N}_{4} \mathrm{O}_{7} \mathrm{~S}, 447$

$\mathrm{C}_{14} \mathrm{H}_{20} \mathrm{ClNO}_{2}, 449$

$\mathrm{C}_{14} \mathrm{H}_{20} \mathrm{Cl}_{2} \mathrm{FeSi}_{2}, 479$

$\mathrm{C}_{14} \mathrm{H}_{27} \mathrm{NO}_{3} \mathrm{Si}, 451$

$\mathrm{C}_{15} \mathrm{H}_{13} \mathrm{Cl}_{2} \mathrm{NO}, 23$

$\mathrm{C}_{15} \mathrm{H}_{13} \mathrm{~N}, 48$

$\mathrm{C}_{15} \mathrm{H}_{13} \mathrm{~N}_{3}, 35$

$\mathrm{C}_{15} \mathrm{H}_{14} \mathrm{Br}_{2} \mathrm{~N}_{4}, 408$

$\mathrm{C}_{15} \mathrm{H}_{14} \mathrm{O}_{3} \mathrm{~S}, 381$ 
$\mathrm{C}_{15} \mathrm{H}_{16}$ NPSSe, 357

$\mathrm{C}_{15} \mathrm{H}_{17} \mathrm{NO}_{2}, 445$

$\mathrm{C}_{15} \mathrm{H}_{18} \mathrm{O}_{6}, 219$

$\mathrm{C}_{15} \mathrm{H}_{22} \mathrm{O}_{4} \mathrm{~S}, 11$

$\mathrm{C}_{16} \mathrm{H}_{20} \mathrm{O}_{5}, 361$

$\mathrm{C}_{16} \mathrm{H}_{26} \mathrm{~N}_{6} \mathrm{Ti}, 477$

$\mathrm{C}_{16} \mathrm{H}_{26} \mathrm{~N}_{8} \mathrm{NiS}_{4}, 156$

$\mathrm{C}_{16} \mathrm{H}_{30} \mathrm{~N}_{4} \mathrm{P}_{2} \mathrm{~S}_{4} \mathrm{Si}_{2}, 399$

$\mathrm{C}_{17} \mathrm{H}_{16} \mathrm{CrO}_{10}, 465$

$\mathrm{C}_{17} \mathrm{H}_{16} \mathrm{O}_{5}, 127$

$\mathrm{C}_{17} \mathrm{H}_{18} \mathrm{ClNO}_{4}, 13$

$\mathrm{C}_{17} \mathrm{H}_{18} \mathrm{~N}_{2} \mathrm{O}_{4} \mathrm{Sn}, 363$

$\mathrm{C}_{17} \mathrm{H}_{20} \mathrm{O}_{8}, 371$

$\mathrm{C}_{17} \mathrm{H}_{21} \mathrm{NO}_{3} \mathrm{~S}, 425$

$\mathrm{C}_{17} \mathrm{H}_{22} \mathrm{O}, 367$

$\mathrm{C}_{17} \mathrm{H}_{25} \mathrm{NO}_{6}, 375$

$\mathrm{C}_{17} \mathrm{H}_{31} \mathrm{NO}_{5}, 7$

$\mathrm{C}_{18} \mathrm{H}_{15} \mathrm{BBr}_{3} \mathrm{P}, 159$

$\mathrm{C}_{18} \mathrm{H}_{16} \mathrm{O}_{3}, 135$

$\mathrm{C}_{18} \mathrm{H}_{16} \mathrm{O} \mathrm{O}_{3} \mathrm{~S}, 257$

$\mathrm{C}_{18} \mathrm{H}_{18} \mathrm{O}_{5}, 473$

$\mathrm{C}_{18} \mathrm{H}_{20} \mathrm{~N}_{2} \mathrm{O}_{2}, 221$

$\mathrm{C}_{18} \mathrm{H}_{23} \mathrm{NO}_{2}$, 441

$\mathrm{C}_{18} \mathrm{H}_{24} \mathrm{FeSi}, 353$

$\mathrm{C}_{18} \mathrm{H}_{26} \mathrm{~N}_{2} \mathrm{O}_{2} \mathrm{Si}, 349$

$\mathrm{C}_{18} \mathrm{H}_{26} \mathrm{O}_{12}, 137$

$\mathrm{C}_{18} \mathrm{H}_{36} \mathrm{AgN}_{3} \mathrm{O}_{9}, 153$

$\mathrm{C}_{18} \mathrm{H}_{45} \mathrm{PSi}_{3}, 249$

$\mathrm{C}_{19} \mathrm{H}_{18} \mathrm{O}_{6}, 385$

$\mathrm{C}_{19} \mathrm{H}_{21} \mathrm{NO}_{6}, 431$

$\mathrm{C}_{19} \mathrm{H}_{25} \mathrm{NO}_{2}, 443$

$\mathrm{C}_{19} \mathrm{H}_{28} \mathrm{Cl}_{2} \mathrm{~N}_{6}, 397$

$\mathrm{C}_{19} \mathrm{H}_{34} \mathrm{NPSSe}, \quad 359$

$\mathrm{C}_{20} \mathrm{H}_{16} \mathrm{~N}_{2} \mathrm{O}_{4}, 389$

$\mathrm{C}_{20} \mathrm{H}_{1}, \mathrm{ClOPRhS}, 273$

$\mathrm{C}_{20} \mathrm{H}_{19} \mathrm{~N}_{4} \mathrm{O}_{9} \mathrm{PV}_{2}, 455$

$\mathrm{C}_{20} \mathrm{H}_{20} \mathrm{O}_{6}, 475$

$\mathrm{C}_{20} \mathrm{H}_{21} \mathrm{ClF}_{3} \mathrm{~N}_{2} \mathrm{O}_{2}, 21$

$\mathrm{C}_{20} \mathrm{H}_{21} \mathrm{~F}_{3} \mathrm{~N}_{2} \mathrm{O}_{2}$, 27

$\mathrm{C}_{20} \mathrm{H}_{22} \mathrm{BrO}_{3} \mathrm{~S}_{16}, 105$

$\mathrm{C}_{20} \mathrm{H}_{24} \mathrm{~N}_{6} \mathrm{O}_{10}, 191$

$\mathrm{C}_{20} \mathrm{H}_{26} \mathrm{~N}_{2} \mathrm{O}_{3} \mathrm{Sn}, 365$

$\mathrm{C}_{20} \mathrm{H}_{28} \mathrm{O}_{10}, 373$

$\mathrm{C}_{21} \mathrm{H}_{20} \mathrm{~N}_{4} \mathrm{OS}, 57$

$\mathrm{C}_{21} \mathrm{H}_{22}, 433,435$

$\mathrm{C}_{21} \mathrm{H}_{24} \mathrm{~N}_{2} \mathrm{O}_{5}, 193$

$\mathrm{C}_{21} \mathrm{H}_{25} \mathrm{Cl}_{2}$ INPSi, 37

$\mathrm{C}_{21} \mathrm{H}_{31} \mathrm{IO}_{8} \mathrm{Si}, 43$

$\mathrm{C}_{21} \mathrm{H}_{32} \mathrm{~B}_{2} \mathrm{~F}_{6} \mathrm{~N}_{4}, 259$

$\mathrm{C}_{22} \mathrm{H}_{14} \mathrm{Br}_{6} \mathrm{CoN}_{2} \mathrm{O}_{2}, 493$

$\mathrm{C}_{22} \mathrm{H}_{17} \mathrm{NO}_{4}, 49$

$\mathrm{C}_{22} \mathrm{H}_{18} \mathrm{~N}_{2} \mathrm{O}_{6} \mathrm{P}_{2} \mathrm{Re}_{2}, 182$

$\mathrm{C}_{22} \mathrm{H}_{24} \mathrm{O}_{2}, 271$

$\mathrm{C}_{22} \mathrm{H}_{27} \mathrm{NO}_{9}, 211$

$\mathrm{C}_{22} \mathrm{H}_{29} \mathrm{NO}_{2}, 203$

$\mathrm{C}_{22} \mathrm{H}_{31} \mathrm{~N}_{2} \mathrm{O}_{4}, 141$

$\mathrm{C}_{23} \mathrm{H}_{16} \mathrm{Cu}_{2} \mathrm{~N}_{3} \mathrm{~S}_{16}, 103$

$\mathrm{C}_{23} \mathrm{H}_{23} \mathrm{NO}_{3} \mathrm{~S}, 223$

$\mathrm{C}_{23} \mathrm{H}_{23} \mathrm{NOS}_{2} \mathrm{Sn}, 285$

$\mathrm{C}_{23} \mathrm{H}_{28} \mathrm{O}_{6}, 131$
$\mathrm{C}_{23} \mathrm{H}_{40} \mathrm{~N}_{4} \mathrm{O}_{8}, 9$

$\mathrm{C}_{23} \mathrm{H}_{44} \mathrm{ClN}_{6} \mathrm{OSi}_{2} \mathrm{Ti}, 481$

$\mathrm{C}_{24} \mathrm{H}_{14} \mathrm{Cl}_{2} \mathrm{~N}_{2} \mathrm{O}_{2} \mathrm{~S}_{2}, 405$

$\mathrm{C}_{24} \mathrm{H}_{16} \mathrm{~S}_{8}, 453$

$\mathrm{C}_{24} \mathrm{H}_{18} \mathrm{CrN}_{2} \mathrm{O}_{5}, 31$

$\mathrm{C}_{24} \mathrm{H}_{20} \mathrm{O}_{4} \mathrm{~S}, 403$

$\mathrm{C}_{24} \mathrm{H}_{26} \mathrm{O}_{6}, 26 \mathrm{l}$

$\mathrm{C}_{24} \mathrm{H}_{40} \mathrm{~N}_{2} \mathrm{O}_{10} \mathrm{~S}, 267$

$\mathrm{C}_{24} \mathrm{H}_{56} \mathrm{I}_{4} \mathrm{~N}_{2} \mathrm{~Pb}, 239$

$\mathrm{C}_{25} \mathrm{H}_{18} \mathrm{AuBrF}_{5} \mathrm{P}, \quad 121$

$\mathrm{C}_{25} \mathrm{H}_{20} \mathrm{~N}_{2} \mathrm{NiO}_{2}, 183$

$\mathrm{C}_{25} \mathrm{H}_{21} \mathrm{Cl}_{3} \mathrm{CrI}, 269$

$\mathrm{C}_{25} \mathrm{H}_{22} \mathrm{ClP}, 335$

$\mathrm{C}_{25} \mathrm{H}_{22} \mathrm{GeO}_{2} \mathrm{~S}$. 379

$\mathrm{C}_{25} \mathrm{H}_{29} \mathrm{NO}_{6}, 209$

$\mathrm{C}_{25} \mathrm{H}_{30} \mathrm{~N}_{2} \mathrm{O}_{5}, 133$

$\mathrm{C}_{25} \mathrm{H}_{30} \mathrm{O}_{8}, 205$

$\mathrm{C}_{26} \mathrm{H}_{24} \mathrm{FeN}_{3} \mathrm{O}_{5}, 125$

$\mathrm{C}_{26} \mathrm{H}_{27} \mathrm{NO}_{4}, 421$

$\mathrm{C}_{26} \mathrm{H}_{29} \mathrm{~N}_{7} \mathrm{O}_{8}, \quad 189$

$\mathrm{C}_{26} \mathrm{H}_{29} \mathrm{NO}_{8}, 207$

$\mathrm{C}_{26} \mathrm{H}_{31} \mathrm{~F}_{3} \mathrm{O}_{2}, 467$

$\mathrm{C}_{26} \mathrm{H}_{36} \mathrm{O}_{6}, 469$

$\mathrm{C}_{26} \mathrm{H}_{44} \mathrm{BCl}_{4} \mathrm{CoF}_{4} \mathrm{~N}_{2}, 437$

$\mathrm{C}_{26} \mathrm{H}_{47} \mathrm{~N}_{2} \mathrm{P}, 253$

$\mathrm{C}_{26} \mathrm{H}_{47} \mathrm{~N}_{2} \mathrm{PS}, 251$

$\mathrm{C}_{27} \mathrm{H}_{22} \mathrm{~N}_{2}, 177$

$\mathrm{C}_{28} \mathrm{H}_{15} \mathrm{Cl}_{8} \mathrm{ErO}_{9}, 109$

$\mathrm{C}_{28} \mathrm{H}_{24} \mathrm{~N}_{2}, 175$

$\mathrm{C}_{28} \mathrm{H}_{25} \mathrm{NO}_{7}, 55$

$\mathrm{C}_{28} \mathrm{H}_{26} \mathrm{~N}_{2} \mathrm{NiP}_{2} \mathrm{~S}_{2}, 165$

$\mathrm{C}_{28} \mathrm{H}_{50} \mathrm{~N}_{2} \mathrm{NiP}_{2} \mathrm{~S}_{2}, \quad 355$

$\mathrm{C}_{29} \mathrm{H}_{35} \mathrm{NO}_{5}, 73$

$\mathrm{C}_{30} \mathrm{H}_{42} \mathrm{~N}_{2} \mathrm{O}_{6}, 139$

$\mathrm{C}_{30} \mathrm{H}_{47} \mathrm{FeNO}_{4} \mathrm{P}_{2}, 245$

$\mathrm{C}_{31} \mathrm{H}_{25} \mathrm{Cl}_{3} \mathrm{~N}_{4} \mathrm{O}_{6} \mathrm{PRh}, 179$

$\mathrm{C}_{31} \mathrm{H}_{42} \mathrm{MoO}_{3} \mathrm{SiW}, 391$

$\mathrm{C}_{31} \mathrm{H}_{48} \mathrm{O}_{3}, 61$

$\mathrm{C}_{31} \mathrm{H}_{50} \mathrm{O}_{3}, 59$

$\mathrm{C}_{32} \mathrm{H}_{25} \mathrm{ClO}_{4} \mathrm{~S}_{2}, 67,69$

$\mathrm{C}_{32} \mathrm{H}_{26} \mathrm{~N}_{8} \mathrm{~S}_{2} \mathrm{Zn}, 333$

$\mathrm{C}_{32} \mathrm{H}_{27} \mathrm{NS}_{2}, 495$

$\mathrm{C}_{32} \mathrm{H}_{40} \mathrm{Cl}_{2} \mathrm{P}_{4} \mathrm{Ru}, 439$

$\mathrm{C}_{32} \mathrm{H}_{44} \mathrm{Cl}_{2} \mathrm{MnN}_{10} \mathrm{O}_{4} \mathrm{~S}_{4}, 19$

$\mathrm{C}_{33} \mathrm{H}_{42} \mathrm{~N}_{4} \mathrm{Si}_{2}, 483$

$\mathrm{C}_{33} \mathrm{H}_{44} \mathrm{BrN}_{5} \mathrm{O}_{8} \mathrm{~S}, 39$

$\mathrm{C}_{33} \mathrm{H}_{57} \mathrm{ClO}_{6} \mathrm{Zr}, 115$

$\mathrm{C}_{34} \mathrm{H}_{26} \mathrm{Cl}_{2} \mathrm{~N}_{4} \mathrm{O}_{4} \mathrm{Ru}, 167$

$\mathrm{C}_{34} \mathrm{H}_{36} \mathrm{O}_{4}, 459$

$\mathrm{C}_{34} \mathrm{H}_{42} \mathrm{CuO}_{4}, 65$

$\mathrm{C}_{34} \mathrm{H}_{42} \mathrm{O}_{10}, 369$

$\mathrm{C}_{34} \mathrm{H}_{62} \mathrm{MoN}_{2} \mathrm{O}_{2} \mathrm{~S}_{4}, 325$

$\mathrm{C}_{35} \mathrm{H}_{34} \mathrm{O}_{7} \mathrm{P}_{2}, 499$

$\mathrm{C}_{35} \mathrm{H}_{54} \mathrm{~N}_{2} \mathrm{O}_{2}, 227$

$\mathrm{C}_{36} \mathrm{H}_{28} \mathrm{Cl}_{6} \mathrm{~N}_{6} \mathrm{O}_{18} \mathrm{Rh}_{2} \mathrm{~S}_{2}, \quad 337$

$\mathrm{C}_{36} \mathrm{H}_{30} \mathrm{SSn}_{2}, 351$

$\mathrm{C}_{36} \mathrm{H}_{50} \mathrm{O}_{16}, 63$

$\mathrm{C}_{36} \mathrm{H}_{58} \mathrm{O}_{4}, 263$

$\mathrm{C}_{36} \mathrm{H}_{62} \mathrm{~N}_{2} \mathrm{O}_{6} \mathrm{PbS}_{2} \mathrm{Si}_{2}, \quad 341$

$\mathrm{C}_{36} \mathrm{H}_{81} \mathrm{AsO}_{9} \mathrm{~S}_{3} \mathrm{Si}_{3}, \quad 343$
$\mathrm{C}_{36} \mathrm{H}_{81} \mathrm{BiO}_{9} \mathrm{~S}_{3} \mathrm{Si}_{3}, \quad 345$

$\mathrm{C}_{36} \mathrm{H}_{81} \mathrm{O}_{9} \mathrm{~S}_{3} \mathrm{SbSi}_{3}, 347$

$\mathrm{C}_{38} \mathrm{H}_{32} \mathrm{P}_{2}, 71$

$\mathrm{C}_{38} \mathrm{H}_{38} \mathrm{Cl}_{4} \mathrm{CoN}_{2} \mathrm{~S}_{2}, \quad 15$

$\mathrm{C}_{38} \mathrm{H}_{44} \mathrm{~N}_{8} \mathrm{O}_{18}, 107$

$\mathrm{C}_{38} \mathrm{H}_{48} \mathrm{LiNO}_{4} \mathrm{P}_{2}, 247$

$\mathrm{C}_{39.6} \mathrm{H}_{69.4} \mathrm{~N}_{8} \mathrm{Si}_{4} \mathrm{Y}, 487$

$\mathrm{C}_{40} \mathrm{H}_{30} \mathrm{~N}_{2}, 173$

$\mathrm{C}_{40} \mathrm{H}_{38} \mathrm{~F}_{6} \mathrm{~N}_{4} \mathrm{O}_{4}, 25$

$\mathrm{C}_{41} \mathrm{H}_{36} \mathrm{O}_{3}, 327$

$\mathrm{C}_{42} \mathrm{H}_{36} \mathrm{CoN}_{3} \mathrm{O}_{3}, 185$

$\mathrm{C}_{42} \mathrm{H}_{3} 7 \mathrm{CuN}_{4} \mathrm{O}_{3} \mathrm{P}_{2}, 275$

$\mathrm{C}_{42} \mathrm{H}_{44} \mathrm{~N}_{2} \mathrm{O}_{4} \mathrm{~S}_{2}, 161$

$\mathrm{C}_{43} \mathrm{H}_{57} \mathrm{~N}_{5} \mathrm{O}_{9}, 111$

$\mathrm{C}_{46} \mathrm{H}_{41} \mathrm{ClP}_{2} \mathrm{Ru}, 501$

$\mathrm{C}_{47} \mathrm{H}_{76} \mathrm{Cl}_{2} \mathrm{~N}_{12} \mathrm{P}_{2} \mathrm{~S}_{6}, \quad 323$

$\mathrm{C}_{48} \mathrm{H}_{116} \mathrm{~N}_{8} \mathrm{Ni}_{4} \mathrm{O}_{82} \mathrm{Zr}_{4}, 171$

$\mathrm{C}_{48} \mathrm{H}_{40} \mathrm{P}_{2} \mathrm{~S}_{17.25} \mathrm{Sb}_{2}, 305$

$\mathrm{C}_{48} \mathrm{H}_{48} \mathrm{O}_{2} \mathrm{Si}_{4}, 149$

$\mathrm{C}_{50} \mathrm{H}_{44} \mathrm{Cl}_{10} \mathrm{GeP}_{26}, 497$

$\mathrm{C}_{52} \mathrm{H}_{60} \mathrm{Ge}_{4}, 146$

$\mathrm{C}_{52} \mathrm{H}_{60} \mathrm{Si}_{4}, 143$

$\mathrm{C}_{54} \mathrm{H}_{82} \mathrm{Hg}_{2} \mathrm{~K}_{2} \mathrm{~N}_{8} \mathrm{O}_{16}, 151$

$\mathrm{C}_{56} \mathrm{H}_{88} \mathrm{Cl}_{8} \mathrm{Co}_{2} \mathrm{~N}_{8} \mathrm{O}_{4}, 17$

$\mathrm{C}_{58} \mathrm{H}_{62} \mathrm{Ag}_{2} \mathrm{~B}_{2} \mathrm{~F}_{8} \mathrm{~N}_{2} \mathrm{O}_{2} \mathrm{P}_{4}, 201$

$\mathrm{C}_{60.5} \mathrm{H}_{63} \mathrm{Cl}_{3} \mathrm{NP}_{4} \mathrm{Pd}_{2}, 409$

$\mathrm{C}_{62} \mathrm{H}_{50} \mathrm{AgO}_{3} \mathrm{P}_{3}, 279$

$\mathrm{C}_{64} \mathrm{H}_{52} \mathrm{~N}_{4} \mathrm{O}_{2} \mathrm{Zn}, 383$

$\mathrm{C}_{80.5} \mathrm{H}_{74} \mathrm{O}_{7.5} \mathrm{P}_{6} \mathrm{Ru}_{3}, 411$

$\mathrm{C}_{82} \mathrm{H}_{62} \mathrm{Fe}_{2} \mathrm{O}_{8} \mathrm{P}_{4}, 157$

$\mathrm{C}_{144} \mathrm{H}_{124} \mathrm{~N}_{8} \mathrm{O}_{12}, 461$

$\mathrm{Cd}_{17} \mathrm{Ga}_{82} \mathrm{~K}_{14} \mathrm{Na}_{21}, 509$

$\mathrm{CsGaTe}_{2}, 91$

$\mathrm{CuH}_{8} \mathrm{O}_{12} \mathrm{Re}_{2}, 294$

$\mathrm{CuHfNaSe}_{3}, 75$

$\mathrm{CuYb}, 508$

$\mathrm{Cu}_{2} \mathrm{~S}_{4}$ TaTI, 94

$\mathrm{Cu}_{2} \mathrm{Te}_{3} \mathrm{Ti}, 93$

$\mathrm{Cu}_{12} \mathrm{Fe}_{13} \mathrm{~S}_{27} \mathrm{Sr}_{6}, 297$

$\mathrm{EuIn}_{2} \mathrm{Ni}, 292$

$\mathrm{F}_{3} \mathrm{H}_{4} \mathrm{NNi}_{2} 3$

FeSi, 320

$\mathrm{Fe}_{2} \mathrm{ScSi}_{2}, 289$

$\mathrm{Ga}_{3} \mathrm{LaO}_{7} \mathrm{Sr}, 76$

Gas Pd, 6

$\mathrm{GdH}_{10} \mathrm{~N}_{3} \mathrm{O}_{14}, 315$

$\mathrm{Ge}_{2}$ In Yb 2,291

$\mathrm{Ge}_{2} \mathrm{Rb}_{4} \mathrm{Se}_{6}, 302$

$\mathrm{HPRb}_{2}, 299$

$\mathrm{H}_{2} \mathrm{~N}_{5} \mathrm{Na}_{2} \mathrm{O}_{16} \mathrm{Pr}, \quad 317$

$\mathrm{H}_{6} \mathrm{LaO}_{15} \operatorname{Re}_{3}, 298$

$\mathrm{H}_{8} \mathrm{NiO}_{12} \mathrm{Re}_{2}, 295$

$\mathrm{H}_{36} \mathrm{Mo}_{7} \mathrm{~N}_{6} \mathrm{O}_{31.5}, 311$

In $_{5} \mathrm{Se}_{8} \mathrm{Tl}, 293$

$\mathrm{K}_{3} \mathrm{~S}_{4} \mathrm{Sb}, 95$

$\mathrm{LiNiO}_{4} \mathrm{P}, 319$

$\mathrm{N}_{9} \mathrm{Na}_{3} \mathrm{Nd}_{2} \mathrm{O}_{27}, 316$

$\mathrm{Na}_{2} \mathrm{Se}_{4}, 87$

$\mathrm{Sc}_{5} \mathrm{Sn}_{3}, 290$

$\mathrm{Te}_{4} \mathrm{~V}_{4.64}, 301$ 